\title{
A regulatory circuit involving miR-143 and DNMT3a mediates vascular smooth muscle cell proliferation induced by homocysteine
}

\author{
HUI-PING ZHANG ${ }^{1 *}$, YAN-HUA WANG ${ }^{1 *}$, CHENG-JIAN CAO $^{2}$, XIAO-MING YANG ${ }^{3}$, \\ SHENG-CHAO MA ${ }^{4}$, XUE-BO HAN ${ }^{5}$, XIAO-LING YANG ${ }^{3}$, AN-NING YANG ${ }^{2}$, \\ JUE TIAN $^{3}$, HUA XU ${ }^{3}$, MING-HAO ZHANG ${ }^{3}$ and YI-DENG JIANG ${ }^{3}$
}

${ }^{1}$ Department of Prenatal Diagnosis Center, General Hospital of Ningxia Medical University, Yinchuan, Ningxia Hui Autonomous Region 750004; ${ }^{2}$ College of Basic and Forensic Medicine, Sichuan University, Chengdu 610041;

${ }^{3}$ Department of Pathophysiology, Basic Medical School, Ningxia Medical University, Yinchuan, Ningxia Hui Autonomous Region 750004; ${ }^{4}$ Department of Physiology,

West China School of Preclinical and Forensic Medicine, Sichuan University, Chengdu 610041;

${ }^{5}$ Department of Laboratory Medicine, Ningxia Medical University, Yinchuan, Ningxia Hui Autonomous, Region 750004, P.R. China

Received December 8, 2014; Accepted September 25, 2015

DOI: $10.3892 / \mathrm{mmr} .2015 .4558$

\begin{abstract}
Accumulating evidence has suggested that homocysteine (Hcy) is an independent risk factor for atherosclerosis (AS). Hcy can promote vascular smooth muscle cell (VSMC) proliferation, which is pivotal in the pathogenesis and progression of AS. The aim of the present study was to investigate the epigenetic regulatory mechanism of microRNA (miR)-143-mediated VSMCs proliferation induced by Hcy. The results of a 3-(4,5-dimethylthiazol-2-yl)-2,5-diphe-nyltetrazolium bromide assay revealed that VSMC proliferation was increased by 1.39 -fold following treatment with $100 \mathrm{mM} \mathrm{Hcy}$, compared with the control group. The levels of miR-143 were markedly downregulated in the Hcy group, compared with the control group, as determined using reverse transcription-quantitative polymerase chain reaction analysis. In addition, the level of miR-143 methylation was increased markedly in the VSMCs treated with Hcy, compared with the control, and was reduced following transfection with DNA methyltransferase (DNMT)3a small interfering RNA, determined using methylation-specific-PCR. The activities of DNMT3a luciferase were also altered accordingly in VSMCs transfected with
\end{abstract}

Correspondence to: Dr Yi-Deng Jiang, Department of Pathophysiology, Basic Medical School, Ningxia Medical University, 1160 Shengli Street, Yinchuan, Ningxia Hui Autonomous Region 750004, P.R. China

E-mail: xuecca@163.com

${ }^{*}$ Contributed equally

Key words: microRNA-143, DNA-methyltransferase 3a, DNA methylation, vascular smooth muscle cell proliferation, homocysteine
pre-miR-143 and miR-143 inhibitor, respectively. In addition, the expression of miR-143 was observed to be inversely correlated with the mRNA and protein expression of DNMT3 in the VSMCs. Taken together, these findings suggest that DNMT3a is a direct target of miR-143, and that the upregulation of DNMT3 is responsible for the hypermethylation of miR-143 in Hcy-induced VSMC proliferation.

\section{Introduction}

Epidemiological and case control studies have consistently indicated that moderate and mild elevation of plasma homocysteine (Hcy), an intermediate metabolite of methionine, is an important and independent risk factor in the development of atherosclerosis (AS) (1-3). Hcy can induce endothelial dysfunction, foam formation and promote vascular smooth muscle cell (VSMC) proliferation (4-6). It is known that VSMC proliferation is pivotal in the pathogenesis and progression of AS, and numerous studies have been performed to elucidate the detailed mechanisms and relevant genes, including platelet-derived growth factor, phosphatase and tensin homolog and $\mathrm{p} 53$, involved in the proliferation of VSMCs (7-9). However, the exact mechanisms that are responsible for Hcy-induced VSMC proliferation remain to be fully elucidated.

MicroRNAs (miRNAs) are short (19-25 nucleotide), non-coding RNAs, which regulate the expression of genes at the post-transcriptional level by binding to the 3 ' untranslated region (UTR) of target mRNAs, leading to mRNA degradation or translation repression (10). Previous evidence has demonstrated that miRNAs are key in the progression of AS, the involvement of which is predominantly associated with controlling the basic function of endothelial cells, VSMCs and macrophages (11). Its expression is modulated by different stimuli involved in every stage of AS. Notably, a group of miRNAs, including miR-143/145, miR-221/222, miR-24, miR-146a and miR-21, have been identified to modulate VSMC 
differentiation, phenotypic switch and neointimal formation following vascular injury $(12,13)$. Among these, miR-143 is a markedly enriched miRNA in VSMCs, which has been investigated extensively in the field of VSMC biology. The expression of miR-143 is critical to the modulation of VSMC phenotype, by promoting differentiation and repressing the proliferation of VSMCs (14). Considerable evidence has shown that the dysregulation of miRNA expression involved in cancer can be triggered by multiple mechanisms, including aberrant DNA methylation of the miRNA promoter (15). However, the underlying mechanisms of miRNA dysregulation in VSMC proliferation resulting from hyperhomocysteinemia remain to be elucidated.

DNA methylation is a major epigenetic factor regulating genome reprogramming, cell differentiation and developmental gene expression, which is catalyzed by a family of DNA methyltransferases (DNMTs), including DNMT1, DNMT3a and DNMT3b $(16,17)$. DNMT1 contributes to the maintenance of DNA methylation patterns, whereas DNMT3a/3b contribute to de novo methylation (18). Hcy is a sulfhydryl-containing amino acid, which is either derived from the metabolic demethylation of methionine or remethylated to methionine (19). Additionally, the hypermethylation of gene promoters is a common mechanism of miRNA silencing at the transcriptional level (20). Previous studies have shown that miRNAs can be involved in the promoter methylation of $\mathrm{CpG}$ islands by targeting the DNMT 3'UTR $(21,22)$. In addition, our previous study found that DNMT3a is one potential target of miR-143 using bioinformatics analysis. Therefore, whether miR-143 methylation regulated by DNMT3a mediates Hcy-induced VSMC proliferation requires clarification.

The present study aimed to identify whether DNA methylation was involved in the downregulation of miR-143 expression and whether there was a circular regulation loop between miR-143 and DNMT3a. The results provided novel insights into the molecular mechanism underlying Hcy-induced VSMC proliferation.

\section{Materials and methods}

Cell culture. Primary cultured VSMCs were obtained from human umbilical vein media (Department of Obstetrics, General Hospital of Ningxia Medical University (Yinchuan, China). The present study was approved by the ethics committee of the Ningxia Medical University. The VSMCs were maintained in Dulbecco's modified Eagle's Medium-Han's F12media (GE Healthcare Life Sciences, Logan, UT, USA) supplemented with $20 \%$ fetal calf serum (FCS; Gibco; Thermo Fisher Scientific, Inc., Waltham, MA, USA), $100 \mathrm{U} / \mathrm{ml}$ penicillin and $100 \mathrm{U} / \mathrm{ml}$ streptomycin (Sigma-Aldrich, St. Louis, MO, USA) at $37^{\circ} \mathrm{C}$ in an incubator with $5 \% \mathrm{CO}_{2}$. The VSMCs were grow for 3-5 generations and seeded into 6-well plates at a density of $1 \times 10^{6}$ cells/well. Hcy (Sigma-Aldrich) was added to the cells at concentrations of 0 (control), 50, 100, 200 and $500 \mu \mathrm{M}$, and $100 \mu \mathrm{M} \mathrm{Hcy}+30 \mu \mathrm{M}$ folate (Sigma-Aldrich), respectively, in order to compensate for the short half-life of homocysteine at $37^{\circ} \mathrm{C}$ in an incubator containing $5 \% \mathrm{CO}_{2}$, which was replenished every $8 \mathrm{~h}$, three times in total.

Cell proliferation assay. Cell proliferation was measured using a 3-(4,5-dimethylthiazol-2-yl)-2,5-diphenyltetrazolium bromide (MTT) assay (Nanjing Jiancheng Bioengineering Institute, Nanjing, China). The VSMCs were seeded in 96-well plates at a density of $10^{3}-10^{4}$ cells/well, and incubated with $0,50,100,200,500 \mu \mathrm{M}$ concentrations of Hcy and $100 \mu \mathrm{M}$ Hcy $+30 \mu \mathrm{M}$ folate for $72 \mathrm{~h}$. Subsequently, $20 \mu \mathrm{l} \mathrm{MTT}$ $(5 \mathrm{mg} / \mathrm{ml})$ was added to each well and incubated at $37^{\circ} \mathrm{C}$ for $4 \mathrm{~h}$. The medium was removed, and $150 \mu \mathrm{l}$ dimethyl sulfoxide (Sigma-Aldrich) was added to each well to resuspend the MTT metabolic product. Following $10 \mathrm{~min}$ of incubation at room temperature, the absorbance of the dissolved formazan was measured at $490 \mathrm{~nm}$ (A490) using a scanning microplate spectrophotometer (BioTek Epoch; BioTek Instruments, Inc., Winooski, VT, USA).

Reverse transcription-quantitative polymerase chain reaction (RT-qPCR) detection of the mRNA expression levels of miR-143 and DNMT3a in VSMCs. Total RNA, containing small RNA, was extracted from the VSMCs using TRIzol reagent (Invitrogen; Thermo Fisher Scientific, Inc.), according to the manufacturer's protocol. For miRNA qPCR, prior RT was performed using a Revertid ${ }^{\text {TM }}$ First Strand cDNA Synthesis kit (Thermo Fisher Scientific, Inc.). For RT, $1 \mu \mathrm{g}$ of RNA containing miRNA was polyadenylated by poly (A) polymerase and then reverse transcribed to cDNA using oligo-dT primers. The cDNA $(2 \mu \mathrm{l})$ then served as the template and was added to $1 \mu 1$ primers for SYBR real-time PCR using TransStart ${ }^{\circledR}$ Top Green qPCR SuperMix (Transgen Biotech Co, Ltd., Beijing, China). The sequence of the miR-143 specific forward primer (Sangon Biotech Co., Ltd., Shanghai, China) was 5'-GGGTGAGATGAAGCACTGTAGCTC-3' and the reverse primer was 5'-GCTGTCAACATACGCTACGTAACG-3'. Human $\mathrm{U}_{6}$ was used for normalization. For DNMT3a mRNA qPCR, cDNA was synthesized with oligo-dT primers and MMLV reverse transcriptase (Promega Corporation, Madison, WI, USA), according to the manufacturer's protocol. GAPDH was used as an endogenous control, and the primer sequences (Sangon Biotech Co., Ltd.) were as follows: DNMT3a, forward 5'-GGGGACGTCCGCAGCGTCACAC-3' and reverse 5'-CAGGGTTGGACTCGAGAAATCGC-3'; and GAPDH, forward 5'-AGAAGGCTGGGGCTCATTTG-3 and reverse 5'-AGGGGCCATCCACAGTCTTC-3'. All qPCR assays were performed using an FTC3000 real-time PCR detection system (FengLing, Shanghai, China) with the following program: 45 cycles at $95^{\circ} \mathrm{C}$ for $45 \mathrm{sec}, 58^{\circ} \mathrm{C}$ for $45 \mathrm{sec}$ and extension $60^{\circ} \mathrm{C}$ for $60 \mathrm{sec}$. The relative quantification of the PCR products was performed according to the $2^{-\Delta \Delta \mathrm{cq}}$ method (23) and normalized by the control.

Western blot analysis. The cultured VSMCs were harvested using a protein extraction kit (Nanjing KeyGen Biotech Co., Ltd., Nanjing, China). A Bicinchoninic Acid kit (Sigma-Aldrich) was used to determine protein concentration. Equal quantities of proteins $(30 \mu \mathrm{g})$ were separated by $10 \%$ SDS-PAGE and then transferred onto a polyvinylidene fluoride membrane (EMD Millipore, Billerica, MA, USA) following which the membrane was blocked in 5\% nonfat milk for $2 \mathrm{~h}$ at room temperature. The membrane was washed following blocking and then incubated overnight at $4^{\circ} \mathrm{C}$ with rabbit polyclonal anti-DNMT3a antibody (1:5,000; Abcam, Cambridge, MA, USA; cat. no. ab23565), and washed three 
time with phosphate-buffered saline with $0.5 \%$ Tween 20 (PBST; pH 4.0) for $5 \mathrm{~min}$ at room temperature. The membranes were then incubated for $2 \mathrm{~h}$ at room temperature with goat anti-rabbit horseradish peroxidase-conjugated IgG secondary antibody $(1: 1,000$; Novoprotein, Shanghai, China; cat. no. AB501). Following washing again three times with PBST, the immunoreactive protein bands were detected using enhanced chemiluminescence solution (Beyotime Institute of biotechnology, China), and the relative protein levels were determined by band densitometry using a gel imaging system (Bio-Rad Laboratories, Inc., Hercules, CA, USA).

Nested methylation-specific (nMS)-PCR for determination of miR-143 methylation in VSMCs. A Wizard ${ }^{\circledR}$ Genomic DNA Purification kit (Promega Corporation) was used to extract GenomicDNA from the VSMCs.AnEZDNAMethylation-Gold kit (Zymo Research, Irvine, CA, USA) was used to integrate DNA denaturation and bisulfite conversion processes. The nMS-PCR consisted of a two-step PCR amplification following standard sodium bisulfite DNA modification. The first step of nMS-PCR was performed using the following outer primer pair: Forward 5'-AGGAGTTTTAGATTAGTTTGGGTAA-3' and reverse 5'-TTTTAAAACAAAATCTCCCTCTATC-3'. The second step of the nMS-PCR was performed using the following PCR primers: Methylation primer, forward 5'-ATTAGTTAGGTATGGTGGTGTACGT-3' and reverse 5'-TTTAAAACAAAATCTCCCTCTATCG-3'; and unmethylation primer, forward 5'-ATTAGTTAGGTATGGTGGTG TATGT-3' and reverse 5'-TTAAAACAAAATCTCCCTCTA TCAC-3'. To reduce mispriming and to increase efficiency, nMS-PCR was used for amplification. A total of $4 \mu 1$ DNA, $12.5 \mu \mathrm{l}$ buffer, and $1 \mu \mathrm{l}$ primers were subjected to 30 cycles in an nMS-PCR program $\left(94^{\circ} \mathrm{C}\right.$ for $30 \mathrm{sec} ; 66^{\circ} \mathrm{C}$ for $30 \mathrm{sec}$ and $72^{\circ} \mathrm{C}$ for $\left.1 \mathrm{~min}\right)$, followed by a $0.5^{\circ} \mathrm{C}$ decrease in the annealing temperature every cycle. Following completion of the nMS-PCR program, 20 cycles were subsequently run $\left(94^{\circ} \mathrm{C}\right.$ for $45 \mathrm{sec}, 51^{\circ} \mathrm{C}$ for $45 \mathrm{sec}$ and $72^{\circ} \mathrm{C}$ for $\left.45 \mathrm{sec}\right)$. Following amplification, $2 \%$ agarose gels containing ethidium bromide (Invitrogen; Thermo Fisher Scientific, Inc.) were used for electrophoresis to separate the PCR products. DNA bands were visualized by ultraviolet light using the Bio-Rad gel imagine system, and the percentage of methylation was calculated using the following formula: Methylation $(\%)=$ methylation $/$ (methylation + unmethylation) $\mathrm{x} 100 \%$.

Cell transfection. The VSMCs were seeded into 6-well plates at a density of $1 \times 10^{6}$ cells/well 1 day prior to transfection. The cells were transiently transfected with pre-miR-143 and an miR-143 inhibitor (Genepharma Co., Ltd., Shanghai, China), DNMT3a-specific small interfering (si)RNA (Genepharma Co., Ltd.) using Lipofectamine 2000 (Invitrogen; Thermo Fisher Scientific, Inc.) according to the manufacturer's protocol. Control-siRNA (Genepharma Co., Ltd.) was co-transfected as a negative control at $37^{\circ} \mathrm{C}$ in an incubator containing $5 \% \mathrm{CO}_{2}$. After $48 \mathrm{~h}$, the cells were collected for total RNA and protein extraction.

Construction of the luciferase reporter vector targeting the DNMT3a 3'-UTR. For the luciferase reporter experiments, a DNMT3a 3'-UTR (Genepharma Co., Ltd.) segment of 611 bp, which contains the potential binding site of miR-143, was amplified using PCR from $2 \mu \mathrm{l}$ human genomic DNA and $1 \mu \mathrm{l}$ primers, and inserted into the pGL3-control vector with the simian virus 40 promoter and enhancer (Promega Corporation) by using the $X b a \mathrm{I}$ site immediately downstream from the stop codon of luciferase. The following sets of primers were used to generate the specific fragments: DNMT3a-UTR, forward 5'-GCTCTAGAACTGGCTACTGCTCTGTG-3'; and reverse 5'-GCTCTAGAGAAATGTATGTCTGTCCCT-3'. The underlined sequences indicate the endonuclease restriction site. The cycling conditions included initial denaturation at $95^{\circ} \mathrm{C}$ for $5 \mathrm{~min}$, followed by 40 cycles of $95^{\circ} \mathrm{C}$ for $10 \mathrm{sec}, 56^{\circ} \mathrm{C}$ for $30 \mathrm{sec}$ and $72^{\circ} \mathrm{C}$ for $30 \mathrm{sec}$, and then extension at $72^{\circ} \mathrm{C}$ for $10 \mathrm{~min}$. When the amplified $3^{\prime}-\mathrm{UTR}$ of DNMT3a, containing the predicted match seed of miR-143, was cloned into the pGL3 vectors, the constructed plasmid, pGL3-DNMT3a, was used to perform the subsequent luciferase assay.

Statistical analysis. GraphPad Prism 5.0 software (GraphPad Software, Inc., La Jolla, CA, USA) was used for statistical analysis. The results are expressed as the mean \pm standard deviation from at least three independent experiments. The data were analyzed using one-way analysis of variance, and were further analyzed using the Student Newman-Keuls test for multiple comparisons within treatment groups or paired Student's t-test for two groups. $\mathrm{P}<0.05$ was considered to indicate a statistically significant difference.

\section{Results}

Expression of miR-143 and its effect on the proliferation of VSMCs induced by Hcy. The effect of Hcy on VSMC proliferation was detected using an MTT assay. As shown in Fig. 1A, VSMC proliferation increased by 1.39 -fold in the $100 \mathrm{mM}$ Hcy group, compared with the control group $(\mathrm{P}<0.05)$. By contrast, VSMC proliferation decreased almost $27 \%$ in the $100 \mu \mathrm{M}$ Hcy+folate group, compared with the $100 \mu \mathrm{M}$ Hcy group $(\mathrm{P}<0.01)$. These results confirmed that Hcy stimulated VSMC proliferation, as reported previously (24), whereas folate exerted a marked antagonistic effect. In addition, the present study detected the expression of miR-143 induced by Hcy in the VSMCs. As shown in Fig. 1B, the levels of miR-143 were significantly downregulated in the Hcy group, particularly in the $100 \mu \mathrm{M}$ Hcy group, compared with the control group $(\mathrm{P}<0.01)$. By contrast, the expression of miR-143 was markedly upregulated in response to $100 \mu \mathrm{M}$ Hcy+folate, compared with the $100 \mu \mathrm{M}$ group $(\mathrm{P}<0.01)$. To further confirm the role of miR-143 in the proliferation of VSMCs induced by Hcy, pre-miR-143 and miR-143 inhibitor were transfected into VSMCs, respectively, to measure cell proliferation. As shown in Fig. 1C, compared with control group, the expression levels of miR-143 were significantly increased in the pre-miR-143 group $(\mathrm{P}<0.01)$, and significantly decreased in the miR-143 inhibitor group $(\mathrm{P}<0.01)$, compared with the control. The proliferation of the VSMCs was increased significantly in the pre-miR-143 transfected cells, whereas the miR-143 inhibitor reduced the levels of VSMC proliferation, compared with the control. In addition, the proliferation of the VSMCs showed a significant increased in the VSMCs treated with $100 \mu \mathrm{M}$ Hcy (Fig. 1D). These results demonstrated that the downregulation 

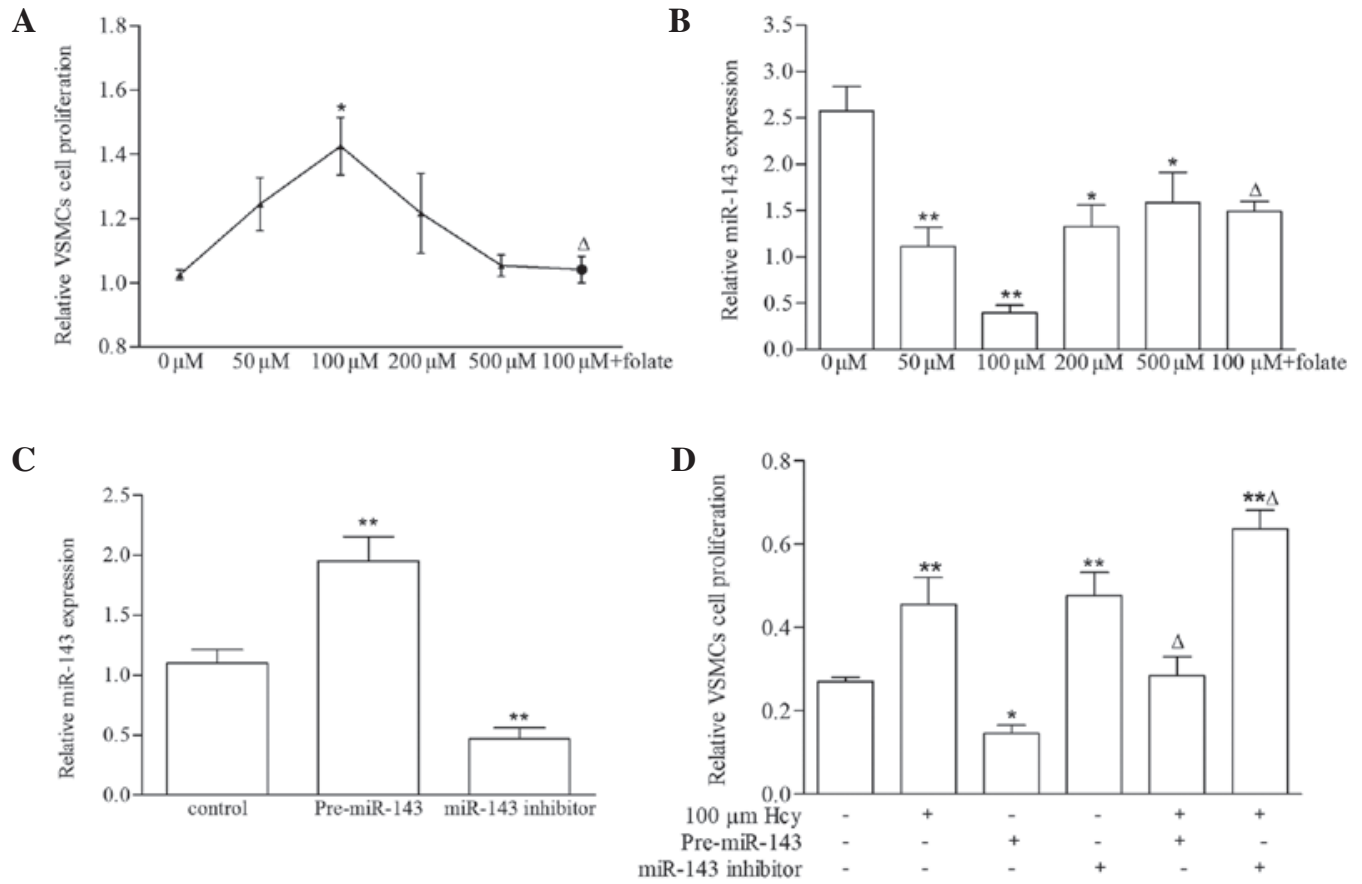

Figure 1. Expression of miR-143 and its effect on the proliferation in VSMCs induced by Hcy. (A) Effect of Hcy on VSMC proliferation, evaluated using an MTT assay. The VSMCs were incubated with 0, 50, 100, 200, $500 \mu \mathrm{M}$ Hcy and $100 \mu \mathrm{M}$ Hcy+30 $\mu \mathrm{M}$ folate for $72 \mathrm{~h}$. (B) Relative levels of miR-143 in VSMCs induced by Hcy were analyzed using reverse transcription-quantitative polymerase chain reaction. Expression of miR-143 was normalized to U6. (C) Relative levels of miR-143 in VSMCs transfected with pre-miR-143 and miR-143 inhibitor. (D) Proliferation of VSMCs transfected with pre-miR-143 and miR-143 inhibitor after $48 \mathrm{~h}$ were measured using an MTT assay. UV-visible absorbance was measured at $490 \mathrm{~nm}$. Data are expressed as the mean \pm standard deviation $(\mathrm{n}=3)$. ${ }^{*} \mathrm{P}<0.05$ and $^{* * *} \mathrm{P}<0.01$, compared with the control group; ${ }^{\mathrm{A}} \mathrm{P}<0.01$, compared with the $100 \mu \mathrm{M}$ Hcy group. miR, microRNA; MTT, 3-(4,5-dimethylthiazol-2-yl)-2,5-diphenyltetrazolium bromide; VSMC, vascular smooth muscle cell; Hcy, homocysteine.

of miR-143 promoted the proliferation of VSMCs induced by Hcy.

Downregulation of miR-143 is caused by DNA hypermethylation in VSMCs induced by Hcy. In order to evaluate the mechanism responsible for the downregulation in the expression of miR-143 in the VSMC, the present study measured the level of methylation in the DNA region encoding miR-143 using nMS-PCR. As shown in Fig. 2, the methylation levels of miR-143 in the 100 and $200 \mu \mathrm{M}$ Hcy groups were significantly increased, by 4.49- and 2.64-fold, respectively, compared with the control group, whereas the methylation levels of miR-143 decreased by $48 \%$ in the $100 \mu \mathrm{M}$ Hcy+folate group, compared with the $100 \mu \mathrm{M}$ Hcy group $(\mathrm{P}<0.01)$. These results indicated that the early downregulation in the production of miR-143 in the Hcy-induced VSMCs was provoked by DNA hypermethylation in the region encoding this microRNA.

DNMT3 a upregulation is responsible for hypermethylation of miR-143 gene promoters in VSMCs induced by Hcy. To investigate whether the hypermethylation of miR-143 was associated with altered expression levels of DNMT3a in VSMCs, the present study determined the expression levels of DNMT3a using RT-qPCR and western blot analyses. As shown in Fig. 3A, the mRNA levels of DNMT3a in the 50, 100 and $200 \mu \mathrm{M}$ Hcy groups were increased by 4.28-, 5.82-, 4.89-fold, respectively, compared with the control group $(\mathrm{P}<0.05)$. By contrast, the mRNA levels of DNMT3a in the $100 \mu \mathrm{M} \mathrm{Hcy}+$ folate group decreased to $45 \%$ of that in the $100 \mu \mathrm{M}$ Hcy group $(\mathrm{P}<0.01)$. In addition, compared with the
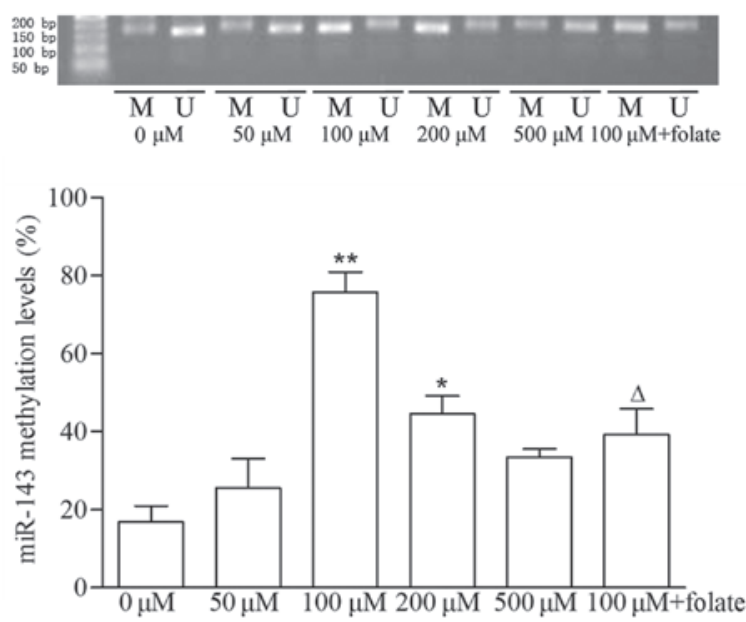

Figure 2. Methylation level of miR-143, detected using nMS-PCR. miR-143 was amplified from bisulfite-converted DNA and analyzed using nMS-PCR. Results represent the mean of three quantifications performed in duplicate, and are expressed as the percentage methylation. Data are expressed as the mean \pm standard deviation $(\mathrm{n}=3)$. ${ }^{*} \mathrm{P}<0.05$ and ${ }^{* *} \mathrm{P}<0.01$, compared with the control group; ${ }^{\Delta} \mathrm{P}<0.01$, compared with the $100 \mu \mathrm{M}$ Hcy group. $\mathrm{M}$, amplified by methylation-specific primer; $\mathrm{U}$, amplified by unmethylation-specific primer; miR, microRNA; VSMC, vascular smooth muscle cell; Hcy, homocysteine; nMS-PCR, nested methylation-specific polymerase chain reaction.

control group, the protein level of DNMT3a in the $100 \mu \mathrm{M}$ Hcy group was significantly increased, by 1.45 -fold, however, in the $100 \mu \mathrm{M}$ Hcy+folate group, the protein expression of DNMT3a decreased to $41 \%$ of that in the $100 \mu \mathrm{M}$ Hcy group $(\mathrm{P}<0.01 ;$ Fig. 3B). To further verify the effect of DNMT3a on 
A

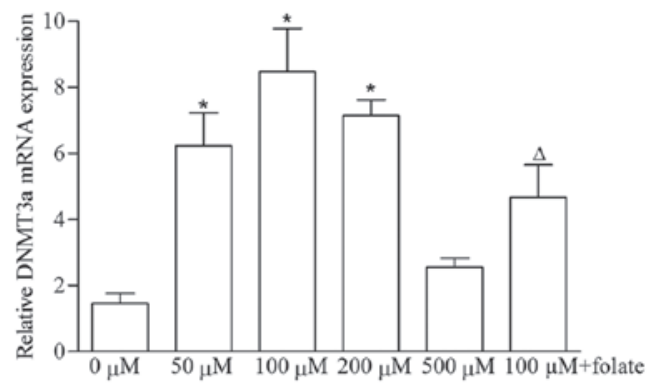

B

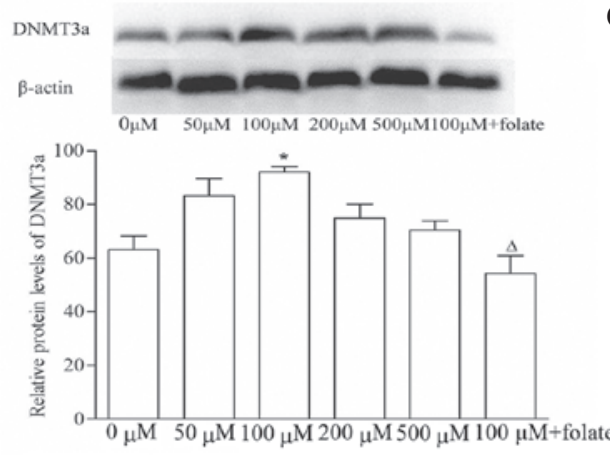

C

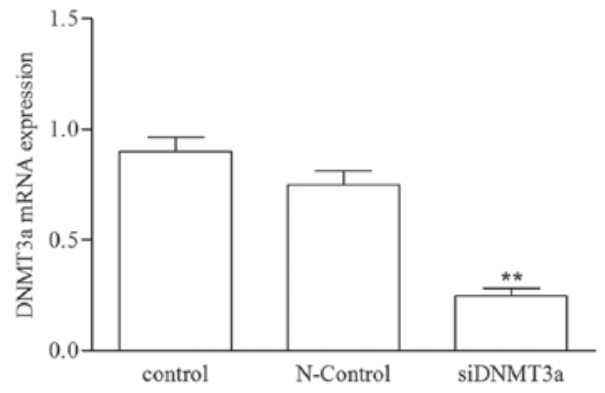

D
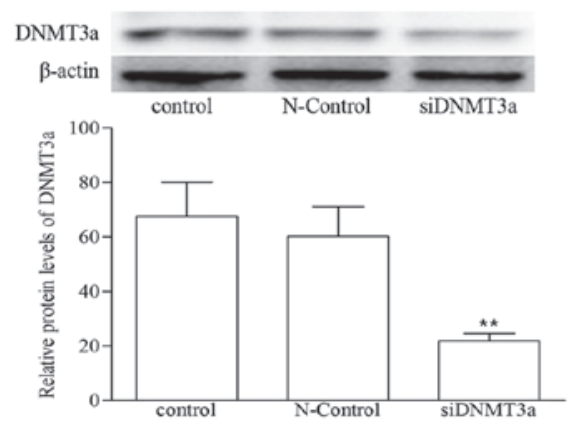

$\mathbf{E}$
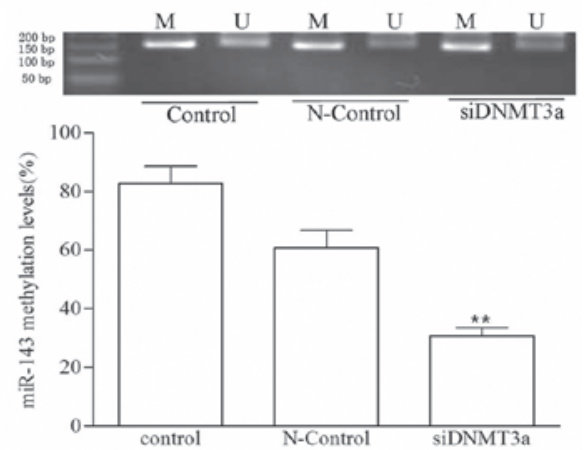

Figure 3. DNMT3a is involved in miR-143 hypermethylation. (A) mRNA levels of DNMT3a in VSMCs were detected using reverse transcription-quantitative PCR, with GAPDH as an internal control. (B) Protein levels of DNMT3a in VSMCs were determined using western blot analysis, with $\beta$-actin as a loading control. The (C) mRNA and (D) protein expression levels of DNMT3a in VSMCs transfected with the DNMT3a siRNA plasmid for $48 \mathrm{~h}$. (E) miR-143 methylation levels in VSMCs following siDNMT3a transfection were analyzed using nested methylation-specific PCR. Data are expressed as the mean \pm standard deviation ( $\mathrm{n}=3$ ). ${ }^{*} \mathrm{P}<0.05$ and $^{* *} \mathrm{P}<0.01$, compared with the control group; ${ }^{\Delta} \mathrm{P}<0.01$, compared with the $100 \mu \mathrm{M}$ Hcy group. M, amplified by methylation-specific primer; U, amplified by unmethylation-specific primer; DNMT, DNA methyltransferase; miR, microRNA; si, small interfering; VSMC, vascular smooth muscle cell; Hcy, homocysteine; PCR, polymerase chain reaction. N-controls, negative controls.

miR-143 hypermethylation, the VSMCs were transfected with a DNMT3a siRNA plasmid for $48 \mathrm{~h}$. The mRNA and protein levels of DNMT3a decreased significantly in the siDNMT3a group, compared with the control or negative controls $(\mathrm{P}<0.01$; Fig. $3 \mathrm{C}$ and $\mathrm{D}$ ). The results suggested a significant reduction in the methylation level of miR-143 following transfection of the VSMCs with DNMT3a siRNA ( $<<0.01$; Fig. 3E), which demonstrated that DNMT3a was important in miR-143 hypermethylation induced by Hcy.

miR-143 targets DNMT3a in VSMCs. In order to identify the possible regulatory pathway of miR-143 targeting DNMT3a in VSMCs, the sequence alignment of human miR-143 with human DNMT3a 3'UTR was conserved (Fig. 4A) (25). The sequence was cloned into the 3'-UTR of the firefly luciferase gene and co-transfected with the pre-miR-143 and miR-143 inhibitor, following which the luciferase activities were assayed using a luciferase reporter system. As shown in Fig. 4B, luciferase activity was significantly reduced in the VSMCs transfected with pre-miR-143, and was increased in the VSMCs transfected with miR-143 inhibitor $(\mathrm{P}<0.01)$. To assess whether the expression of DNMT3a was regulated by miR-143, RT-qPCR and western blot analyses were performed to determine the mRNA and protein levels of DNMT3a following transfection of the VSMCs with the pre-miR-143 and miR-143 inhibitor. As shown in Fig. 4C and D, enforced miR-143 expression led to a reduction in the expression of DNMT3a at the mRNA and protein levels, compared with the control group. By contrast, the inhibition of miR-143 in the VSMCs increased the expression of DNMT3a. These results demonstrated that miR-143 directly targeted DNMT3a by binding their 3'-UTRs, thus suppressing protein expression.

\section{Discussion}

Accumulating evidence has suggested that Hcy is an independent risk factor of AS, although the underlying mechanism remains to be fully elucidated (26). In particular, evidence has 
A
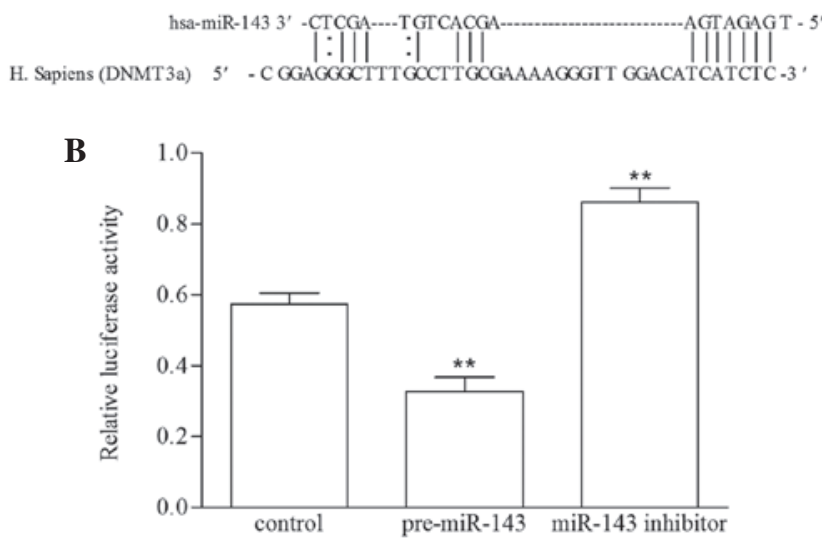

C

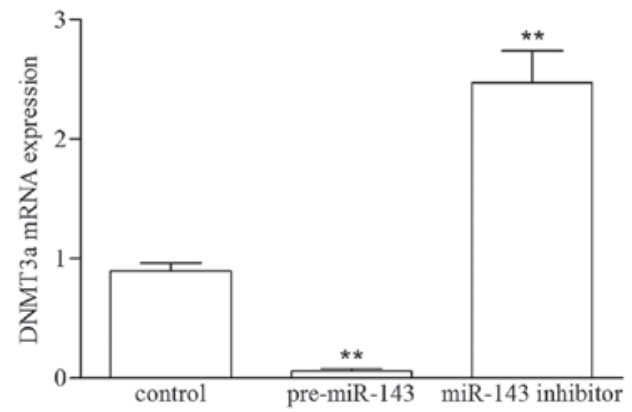

D
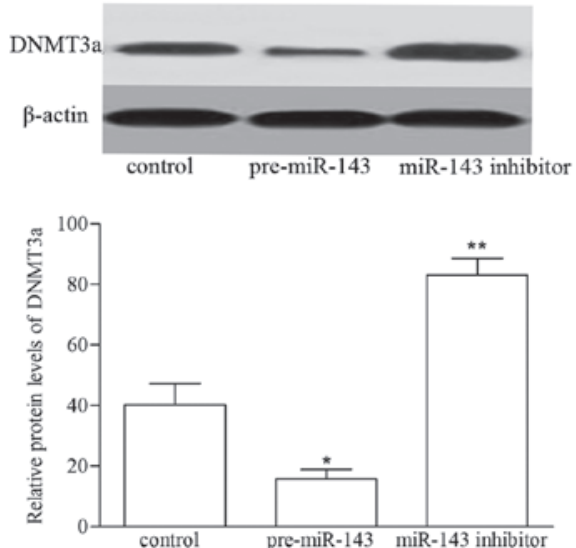

Figure 4. miR-143 directly targets DNMT3a in VSMCs. (A) Sequence alignment of human miR-143 (bold) matches the 3' untranslated region of DNMT3a. (B) Relative luciferase activities were analyzed in VSMCs transfected with pre-miR-143 and miR-143 inhibitor for $48 \mathrm{~h}$. Relative (C) mRNA and (D) protein levels of DNMT3a in VSMCs transfected with pre-miR-143 and miR-143 inhibitor were detected using reverse transcription-quantitative polymerase chain reaction and western blot analyses, respectively. Data are expressed as the mean \pm standard deviation $(\mathrm{n}=3)$. ${ }^{*} \mathrm{P}<0.05$ and ${ }^{* *} \mathrm{P}<0.01$, compared with the control group. DNMT, DNA methyltransferase; miR, microRNA; VSMC, vascular smooth muscle cell; Hcy, homocysteine.

indicated that the proliferation of VSMCs is important in the development of AS (27). The present study showed that Hcy had a stimulatory effect on VSMC proliferation, which was consistent with these previous studies. However, the underlying mechanism accounting for the promotion of VSMC proliferation by Hcy remains to be fully elucidated.

In previous years, emerging evidence has demonstrated that miRNAs are pivotal in the control of VSMC proliferation and differentiation (28). Several studies have demonstrated that miR-143 is involved in the phenotypic modulation of VSMCs and cardiovascular diseases $(29,30)$. The results obtained from RT-qPCR validation in the present study showed that miR-143 was downregulated in VSMCs induced by Hcy, particularly at a concentration of $100 \mu \mathrm{M}$ Hcy, which exhibited the most marked proliferating effect on the VSMCs. In addition, the VSMCs transfected pre-miR-143 and miR-143 inhibitor further confirmed the suppressive effect of miR-143 on VSMC proliferation, which had the same effect as folate. In the 'methionine cycle', Hcy can be converted to methionine by acquiring a methyl group from N-5-methyltetrahydrofolate by methionine synthase, and be subsequently activated to S-adenosyl methionine (SAM) (31). Folate supply increases the production of $\mathrm{N}-5$-methyltetrahydrofolate, promoting the transformation of Hcy to SAM (32). Therefore, the addition of folate in the present study had an antagonistic effect on the deteriorative roles of Hcy. In the present study, the expression of miR-143 was downregulated in the Hcy group, which attenuated its suppressive effect and promoted VSMC proliferation.

Increasing evidence has shown that DNA hypermethylation is one cause of inactivation for numerous genes, and has been identified more recently as a cause of the inactivation of miRNA gene expression (33). Hcy is an intermediate product in transmethylation reactions, including DNA methylation modification, which is involved in the epigenetic regulation of gene expression (34). Therefore, the present study further evaluated the mechanism responsible for the downregulated expression of miR-143 in the VSMCs. As the results of the nMS-PCR analysis revealed, there was an increase in the level of methylation in the miR-143-coding region, however, its methylation level was decreased in the folate group, due to the reverse effects of folate against Hcy. Combining the effects of folate on the methylation status of miR-143 further supported the role of miR-143 in VSMC proliferation. As DNA hypermethylation inhibits gene expression (35), the results of the present study demonstrated that aberrant miR-143 DNA hypermethylation was responsible for the downregulation in the expression of miR-143 in Hcy-induced VSMCs.

It is well supported that altered gene-specific methylation triggered by abnormal functioning of DNMTs may cause the transcriptional silencing of cancer-associated genes in human cancer (36). Therefore, aberrant gene-specific methylation changes in VSMCs prompted the present study to investigate 
whether these changes are associated with altered expression of DNMTs in VSMCs. DNMT3a is responsible for setting genomic DNA methylation patterns, a key layer of epigenetic information (37). It has been previously demonstrated that miR-143 targets DNMT3a mRNA (30). In order to verify whether DNMT3a mediates the miR-143 DNA hypermethylation process, the present study examined the expression of DNMT3a in VSMCs induced by Hcy. The results showed that the mRNA and protein levels of DNMT3a were significantly upregulated in the VSMCs, and were downregulated in the folate group. A similar previous study demonstrated that DNMT3a mRNA levels was high in breast tumors, accompanied by DNA hypermethylation (38). In addition, miR-143 methylation levels in the present study were decreased following transfection of the VSMCs with the DNMT3a siRNA plasmid, which further verified that DNMT3a was important in miR-143 hypermethylation. However, the mechanism responsible for DNMT3a upregulation remain to be fully elucidated

It has been previously demonstrated that certain microRNAs targeting DNMTs transcripts lead to the demethylation and transcriptional activation of numerous protein coding gene sequences, thereby contributing to gene expression (39). In the present study, the upregulation of DNMT3a in VSMCs was accompanied by an overall decrease in the expression of miRNA-143 in the VSMCs induced by Hcy, which suggested that miR-143 may control the functioning of DNMT3a. Notably, using bioinformatics analysis, our previous study found that DNMT3a is one potential target of miR-143 (30). To confirm whether DNMT3a is the direct target of miR-143 in VSMCs in the present study, pre-miR-143 and a miR-143 inhibitor were co-transfected with the recombinant plasmid containing the DNMT3a complementary site. The luciferase activity of DNMT3a were negatively correlated with the levels of miR-143 levels in the VSMCs transfected with the pre-miR-143 and inhibitor. Similarly, an inverse correlation was observed between the expression of miR-143 and DNMT3a in the VSMCs, further confirming that miR-143 negatively regulated DNMT3a.

In conclusion, the data of the present study demonstrated for the first time, to the best of our knowledge, that the upregulation of DNMT3a directly results in the hypermethylation of miR-143 genes, leading to a downregulation in the expression of miR-143 expression, accompanied by VSMC proliferation. These results demonstrate a novel regulatory circuit between DNMT3a and miR-143 in VSMC proliferation.

\section{Acknowledgements}

This study was supported by grants from the National Natural Science Foundation of China (grant. no. 1260105, 81360053, 81360027 and 81200118), the Ningxia Education Department Scientific and Technological Project (grant nos. NGY2013054 and NGY2013082) and the Ningxia Science and Technique Project (grant no. NZ14120).

\section{References}

1. van den Brandhof WE, Haks K, Schouten EG and Verhoef P. The relation between plasma cysteine, plasma homocysteine and coronary atherosclerosis. Atherosclerosis 157: 403-9, 2001.
2. Ozkan Y, Ozkan E and Simşek B: Plasma total homocysteine and cysteine levels as cardiovascular risk factors in coronary heart disease. Int J Cardio 82: 269-277, 2002.

3. Zhang D, Wen X, Wu W, Xu E, Zhang Y and Cui W: Homocysteine-related hTERT DNA demethylation contributes to shortened leukocyte telomere length in atherosclerosis. Atherosclerosis 231: 173-179, 2013.

4. Horvath B, Szapary L, Debreceni L, Feher G, Kenyeres P, Fulop A, Battyani I and Toth K: Effect of Sclerovit on endothelial dysfunction, hemorheological parameters, platelet aggregation, plasma concentration of homocysteine and progression of atherosclerosis in patients with vascular diseases. Clin Hemorheol Microcirc 42: 19-28, 2009.

5. Liang Y, Yang X, Ma L, Cai X, Wang L, Yang C, Li G, Zhang M, Sun W and Jiang Y: Homocysteine-mediated cholesterol efflux via ABCA1 and ACAT1 DNA methylation in THP-1 monocyte-derived foam cells. Acta Biochim Biophys Sin (Shanghai) 45: 220-228, 2013.

6. Jacob T, Hingorani A and Ascher E: Evidence for telomerase activation in VSMCs exposed to hyperglycemic and hyperhomocysteinemic conditions. Angiology 60: 562-568, 2009.

7. Kim Y,Han JH, Yun E, Jung SH, Lee JJ, Song GY and Myung CS: Inhibitory effect of a novel naphthoquinone derivative on proliferation of vascular smooth muscle cells through suppression of platelet-derived growth factor receptor $\beta$ tyrosine kinase. Eur J Pharmacol 733: 81-89, 2014.

8. Huang $\mathbf{J}$ and Kontos CD: Inhibition of vascular smooth muscle cell proliferation, migration and survival by the tumor suppressor protein PTEN. Arterioscler Thromb Vasc Biol 22: 745-751, 2002.

9. Yonemitsu Y, Kaneda Y, Tanaka S, Nakashima Y, Komori K, Sugimachi K and Sueishi K: Transfer of wild-type p53 gene effectively inhibits vascular smooth muscle cell proliferation in vitro and in vivo. Circ Res 82: 147-156, 1998.

10. Bartel DP: MicroRNAs: Genomics, biogenesis, mechanism, and function. Cell 116: 281-297, 2004.

11. Zhang C: MicroRNA and vascular smooth muscle cell phenotype: New therapy for atherosclerosis?. Genome Med 1: 85, 2009.

12. Rangrez AY, Massy ZA, Metzinger-Le Meuth V and Metzinger L: MiR-143 and miR-145: Molecular keys to switch the phenotype of vascular smooth muscle cells. Circ Cardiovasc Genet 4: 197-205, 2011.

13. Dong S, Xiong W, Yuan J, Li J, Liu J and Xu X: MiRNA-146a regulates the maturation and differentiation of vascular smooth muscle cells by targeting NF- $\mathrm{kB}$ expression. Mol Med Rep 8: 407-412, 2013.

14. Boucher JM, Peterson SM, Urs S, Zhang C and Liaw L: The miR-143/145 cluster is a novel transcriptional target of Jagged-1/Notch signaling in vascular smooth muscle cells. J Biol Chem 286: 28312-28321, 2011.

15. Saetrom P, Snøve O Jr and Rossi JJ: Epigenetics and microRNAs. Pediatr Res 61: 17R-23R, 2007.

16. Hutnick LK, Golshani P, Namihira M, Xue Z, Matynia A, Yang XW, Silva AJ, Schweizer FE and Fan G: DNA hypomethylation restricted to the murine forebrain induces cortical degeneration and impairs postnatal neuronal maturation. Hum Mol Genet 18: 2875-2888, 2009.

17. Liu K, Wang YF, Cantemir C and Muller MT: Endogenous assays of DNA methyltransferases: Evidence for differential activities of DNMT1, DNMT2 and DNMT3 in mammalian cells in vivo. Mol Cell Biol 23: 2709-2719, 2003.

18. Arand J, Spieler D, Karius T, Branco MR, Meilinger D, Meissner A, Jenuwein T, Xu G, Leonhardt H, Wolf V and Walter J: In vivo control of $\mathrm{CpG}$ and non-CpG DNA methylation by DNA methyltransferases. PLoS Genet 8: e1002750, 2012.

19. Krebs HA, Hems R and Tyler B: The regulation of folate and methionine metabolism. Biochem J 158: 341-353, 1976.

20. Toyota M, Suzuki H, Sasaki Y, Maruyama R, Imai K, Shinomura Y and Tokino T: Epigenetic silencing of microRNA-34b/c and B-cell translocation gene 4 is associated with $\mathrm{CpG}$ island methylation in colorectal cancer. Cancer Res 68: 4123-4132, 2008.

21. Veeck J and Esteller M: Breast cancer epigenetics: From DNA methylation to microRNAs. J Mammary Gland Biol Neoplasia 15: 5-17, 2010.

22. Robaina MC, Mazzoccoli L, Arruda VO, Reis FR, Apa AG, de Rezende LM and Klumb CE: Deregulation of DNMT1, DNMT3B and miR-29s in Burkitt lymphoma suggests novel contribution for disease pathogenesis. Exp Mol Pathol 98: 200-207, 2015. 
23. Rao X, Huang X, Zhou Z and Lin X: An improvement of the $2^{\wedge}$ (-delta delta CT) method for quantitative real-time polymerase chain reaction data analysis. Biostat Bioinforma Biomath 3: 71-85, 2013.

24. Yideng J, Jianzhong Z, Ying H, Juan S, Jinge Z, Shenglan W, Xiaoqun $\mathrm{H}$ and Shuren W: Homocysteine-mediated expression of SAHH, DNMTs, MBD2 and DNA hypomethylation potential pathogenic mechanism in VSMCs. DNA Cell Biol 26: 603-611, 2007.

25. Ng EK, Tsang WP, Ng SS, Jin HC, Yu J, Li JJ, Röcken C, Ebert MP, Kwok TT and Sung JJ: MicroRNA-143 targets DNA methyltransferases 3A in colorectal cancer. Br J Cancer 101: 699-706, 2009.

26. Pawlak K, Mysliwiec M and Pawlak D: Hyperhomocysteinemia and the presence of cardiovascular disease are associated with kynurenic acid levels and carotid atherosclerosis in patients undergoing continuous ambulatory peritoneal dialysis. Thromb Res 129:704-9, 2012

27. Chistiakov DA, Orekhov AN and Bobryshev YV: Vascular smooth muscle cell in atherosclerosis. Acta Physiol (Oxf) 214: 33-50, 2015.

28. Choe N, Kwon JS, Kim JR, Eom GH, Kim Y, Nam KI, Ahn Y, Kee HJ and Kook H: The microRNA miR-132 targets Lrrfipl to block vascular smooth muscle cell proliferation and neointimal hyperplasia. Atherosclerosis 229: 348-355, 2013.

29. Davis-Dusenbery BN, Chan MC, Reno KE, Weisman AS Layne MD, Lagna $\mathrm{G}$ and Hata A: down-regulation of Kruppel-like factor-4 (KLF4) by microRNA-143/145 is critical for modulation of vascular smooth muscle cell phenotype by transforming growth factor-beta and bone morphogenetic protein 4. J Biol Chem 286 : 28097-28110, 2011

30. Zhao W, Zhao SP and Zhao YH: MicroRNA-143/-145 in cardiovascular diseases. Biomed Res Int 2015: 531740, 2015.

31. Isa $Y$, Mishima $T$, Tsuge $H$ and Hayakawa $T$ : Increase in $\mathrm{S}$-adenosylhomocysteine content and its effect on the S-adenosylhomocysteine hydrolase activity under transient high plasma homocysteine levels in rats. J Nutr Sci Vitaminol (Tokyo) 52: 479-482, 2006
32. Smith DE, Smulders YM, Blom HJ, Popp J, Jessen F, Semmler A, Farkas M and Linnebank M: Determinants of the essential one-carbon metabolism metabolites, homocysteine, $\mathrm{S}$-adenosylmethionine, $\mathrm{S}$-adenosylhomocysteine and folate, in cerebrospinal fluid. Clin Chem Lab Med 50: 1641-1647, 2012.

33. Sandhu R, Rivenbark AG, Mackler RM, Livasy CA and Coleman WB: Dysregulation of microRNA expression drives aberrant DNA hypermethylation in basal-like breast cancer. Int J Oncol 44: 563-572, 2014.

34. Ma S, Zhang H, Sun W, Gong H, Wang Y, Ma C, Wang J, Cao C, Yang X, Tian J and Jiang Y: Hyperhomocysteinemia induces cardiac injury by up-regulation of p53-dependent Noxa and Bax expression through the p53 DNA methylation in ApoE(-/-) mice. Acta Biochim Biophys Sin (Shanghai) 45: 391-400, 2013

35. Magdinier F, Billard LM, Wittmann G, Frappart L, Benchaïb M, Lenoir GM, Guérin JF and Dante R: Regional methylation of the $5^{\prime}$ end $\mathrm{CpG}$ island of BRCA1 is associated with reduced gene expression in human somatic cells. FASEB J 14: 1585-1594, 2000.

36. He M, Fan J, Jiang R, Tang WX and Wang ZW: Expression of DNMTs and genomic DNA methylation in gastric signet ring cell carcinoma. Mol Med Rep 8: 942-948, 2013.

37. Holz-Schietinger C, Matje DM and Reich NO: Mutations in DNA methyltransferase (DNMT3A) observed in acute myeloid leukemia patients disrupt processive methylation. J Biol Chem 287: 30941-30951, 2012.

38. Yu Z, Xiao Q, Zhao L, Ren J, Bai X, Sun M, Wu H, Liu X, Song Z, Yan Y, et al: DNA methyltransferase 1/3a overexpression in sporadic breast cancer is associated with reduced expression of estrogen receptor-alpha/breast cancer susceptibility gene 1 and poor prognosis. Mol Carcinog, 2014.

39. Sun X, He Y, Huang C, Ma TT and Li J: The epigenetic feedback loop between DNA methylation and microRNAs in fibrotic disease with an emphasis on DNA methyltransferases. Cell Signal 25: 1870-1876, 2013. 